Revue d'histoire de l'Amérique française

REVUE D.HISTOIRE DE L'AMÉRIQUE FRANÇAISE

\title{
Position de la Nouvelle-France en 1663
}

\section{Gustave Lanctot}

Volume 11, numéro 4, mars 1958

URI : https://id.erudit.org/iderudit/301861ar

DOI : https://doi.org/10.7202/301861ar

Aller au sommaire du numéro

Éditeur(s)

Institut d'histoire de l'Amérique française

ISSN

0035-2357 (imprimé)

1492-1383 (numérique)

Découvrir la revue

Citer cet article

Lanctot, G. (1958). Position de la Nouvelle-France en 1663. Revue d'histoire de l'Amérique française, 11(4), 517-532. https://doi.org/10.7202/301861ar d'utilisation que vous pouvez consulter en ligne.

https://apropos.erudit.org/fr/usagers/politique-dutilisation/ 


\section{POSITION DE LA NOUVELLE-FRANCE EN 1663 *}

L'année 1663 ferme la première période de l'histoire de la Nouvelle-France, qui va des découvertes à la suppression des compagnies de commerce. Elle forme un haut lieu, qui permet une vue rétrospective de l'effort colonial et des résultats acquis au milieu du dix-septième siècle.

A cette date, le territoire exploré par les Français se révèle immense, englobant un cinquième de la terre nord-américaine, du Cap-Breton et de Terre-Neuve au Mississipi, et du Labrador et de la baie James au lac Érié. Prestigieux par son ampleur, le fleuve Saint-Laurent permet de pénétrer, sur ses eaux et celles de l'Outaouais, jusqu'aux Grands Lacs et au delà, pendant que ses affluents, le Saguenay et le Saint-Maurice, ouvrent des voies vers le Nord, et que la Chaudière et le Richelieu offrent leur route vers le Sud. Dans cette immensité, le territoire peuplé se limite le long du fleuve, du Saguenay au Saut Saint-Louis, à trois postes, Québec, les Trois-Rivières et Montréal, isolés les uns des autres par d'interminables étendues de hautes forêts, et sa population totale atteint à peine deux mille cinq cents âmes.

Porte d'entrée du pays, Tadoussac, que les gros navires préférèrent longtemps ne point dépasser, n'était plus qu'un port d'escale et de transbordement dans les cas de retours pressés. Dans la saison, les barques de Québec venaient y troquer avec les Montagnais, que les Jésuites évangélisaient. A côté de son fortin et de sa petite chapelle, quelques cabanes servaient de logements, dont les occupants bénéficiaient de deux moulins à eau. Il fallait de là, remonter environ quatre-vingt-dix milles avant de rencontrer les premières habitations du Cap Tourmente. Ensuite, quel-

* Ce texte forme le premier de deux chapitres rétrospectifs d'une Histoire du Canada des origines à l'administration royale, prête à passer aux mains de l'éditeur. 
que dix milles plus loin, commençaient à s'échelonner, sur une étendue de huit lieues, les maisons des seigneuries de Beaupré, de Beauport et de l'île d'Orléans, tandis que, celles de Notre-Damedes Anges, sur la rivière Saint-Charles, et de Lauson, sur la rive sud du fleuve, s'alignaient à la vue de Québec. A peu de distance de ce poste, sur sa droite, se groupaient les habitations du coteau Sainte-Geneviève et, sur le fleuve, deux milles plus loin, celles de Sillery et neuf milles en amont celles du Cap Rouge. Toute cette étendue de pays d'environ trente milles, avec chaque maison sur sa propre terre, formait le noyau principal de la colonie et sa population pouvait se chiffrer à près de neuf cents âmes.

Dans un paysage grandiose, escaladant un promontoire en bordure de son large fleuve, la petite capitale de Québec offrait soudain au regard son groupement pittoresque. Au rebord abrupt du Cap Diamant, s'érigeait, résidence du gouverneur, son fort Saint-Louis aux embrasures pointant leurs canons dans l'espace. Sur le bleu foncé du ciel, se profilaient les flèches de ses petites églises en pierre: la cathédrale et les chapelles des Jésuites, des Ursulines et des Hospitalières, pendant qu'à quelque distance sur la droite, au-dessus des blés voisins, tournaient dans le soleil, les ailes blanches d'un moulin à vent. Adjoignant le fort, la Place d'Armes bordait la spacieuse maison de la Compagnie de la Nouvelle-France, où siégeaient le conseil du pays et la cour de la sénéchaussée. En face, s'élevait la cathédrale que la Grande Place séparait de la maison et du collège des Jésuites. Autour de ce centre de la ville haute, s'espaçaient, avec leurs jardins, le couvent des Ursulines, l'Hôtel-Dieu des Hospitalières, quelques résidences en pierres meulières, et de modestes maisons de bois. A gauche du chemin qui descendait à la basse-ville, les Hurons avaient élevé une enceinte de pieux entourant leurs tentes de bouleaux, où une centaine d'entre eux vivaient à l'indienne de chasse et de pêche. Le long du port, que n'osait dépasser aucun navire, la basse-ville comprenait les boutiques, les magasins et les hangars, ainsi que les maisons des principaux marchands, toutes de pierre et les mieux bâties de la ville. La population de Québec s'élevait à près de huit cents âmes. 
Au delà du Cap-Rouge, le long du fleuve que bordaient d'épaisses forêts, il fallait remonter sur une distance d'environ soixante-et-dix milles avant d'apercevoir les habitations de la seigneurie du Cap-de-la-Madeleine et de parvenir, trois milles plus loin, aux Trois-Rivières. Pourvu d'un gouverneur particulier, possédant une cour de justice et une église en bois, ce poste tirait son importance de la grande foire annuelle des fourrures. A l'intérieur de son enceinte de pieux, ses maisons et ses quelques boutiques abritaient environ deux cent cinquante personnes.

Passé les Trois-Rivières, la forêt ubiquitaire, haute et drue, couvrait les deux rives du fleuve jusqu'à Montréal, dernière limite des habitations. Ses quelque cinquante maisons se rangeaient les unes à la vue des autres, depuis le fort de la Pointe Callières jusqu'au bas de la Place d'Armes, pendant que, plus loin, sur une éminence, aujourd'hui disparue, en face du square Viger, s'élevaient un fortin et un moulin à vent. Au bord d'un vaste élargissement du fleuve et aux pieds de la verdoyante forêt de son Mont-Royal, le poste de la seigneurie de Montréal offrait un agréable coup d'œil, avec sa petite église en bois, son Hôtel-Dieu et les spacieuses résidences du séminaire de Saint-Sulpice et de M. Le Moyne. Dernier né des postes, ne possédant encore qu'une école pour les petits garçons et les petites filles, Montréal avait si bien grandi qu'il se targuait de grouper déjà près de cinq cents habitants.

En total, deux mille cinq cents habitants, voilà tout ce que la Nouvelle-France, de Tadoussac à Montréal, sur une étendue de deux cent soixante milles en bordure du Saint-Laurent, renfermait après cinquante-cinq ans de colonisation. Ce chiffre s'accorde assez bien, pour qu'on le maintienne, avec la statistique de l'émigration et des naissances. Approximativement, ce total pouvait se récapituler comme suit: Québec, 800 ; Montréal, 500; Trois-Rivières, 250 ; Cap-de-la-Madeleine, 50 ; campagnes de Québec, du Cap Tourmente au Cap Rouge, 900; total: 2,500 âmes.

Cette petite population sortait, en nombre fortement inégal, des trente-cinq provinces de la France contemporaine. Pour la période de 1608 à 1660, la statistique indique l'origine de ces 
émigrants, dont elle fixe le chiffre à douze cent soixante. Laissant de côté celles d'où vinrent moins de vingt-cinq d'entre eux, voici les dix provinces qui ont fourni les plus forts effectifs: Normandie, 359 ; Perche, 211; Aunis, 138; Ille-de-France, 112; Maine, 67; Anjou, 58; Poitou, 54; Saintonge, 47; Beauce, 36; Champagne, 30 , soit un total de 1151 colons sur 1260 . Un coup d'œil à une carte de l'ancienne France montre que ces provinces peuvent se ramener à trois grandes régions: celle du Nord, joignant la Normandie et le Perche, avec 637 colons; celle de l'Ouest, groupant le Maine, l'Anjou, le Poitou, l'Aunis et la Saintonge, avec 364, et celle du Centre-Nord, formé de l'Île-de-France, de la Beauce et de la Champagne, avec 178.

Les premiers arrivés sont les Percherons, qui viennent dès le retour de Champlain, et les Normands, qui profitent des embarquements des armateurs de Rouen. Avec Nicolet, Tilly, Repentigny et Le Moyne, ils marqueront leur nouvelle patrie de leur audace maritime, pendant que Giffard, Boucher, Maheux et Gagnon la consolideront par leur ténacité terrienne. Venus plus tard, recrutés par La Dauversière, qui est de La Flèche, les Angevins et les Poitevins, auxquels se joindront les Aunisiens, tentés par les départs de La Rochelle, s'installeront plutôt à Montréal. Formés à ce séculaire carrefour de batailles, ils seront les combatifs et les obstinés qui barreront la route aux Iroquois. Quant au groupe de l'île-de-France, de la Beauce et de la Champagne, le moins nombreux, il exerça surtout une influence sociale avec Godefroy, d'Amours, de Lotbinière, d'Auteuil, Jeanne Mance et Marguerite Bourgeoys, qui furent des notabilités ou des animateurs. Au cours de cette période de peuplement, ce sont les provinces situées au nord d'une ligne diagonale allant de la Picardie à la Saintonge, qui ont fourni la quasi-totalité des colons, soit 1112, pendant que celles de l'Est et du Sud ne contribuèrent chacune qu'un nombre insignifiant, soit un total de 118 personnes à partager entre vingt-cinq provinces. Ainsi ce sont les hommes du Nord-Ouest qui seront les ancêtres du peuple canadien, en même temps qu'ils deviendront les formateurs de la forte émigration qui suivra leurs traces après 1663 . 
Il reste à savoir à quelles couches sociales appartenaient ces pionniers de la première heure. La réponse s'en présente facilement. Avant 1633, Québec ne reçut aucun colon, n'abritant dans son «Habitation » que des interprètes, des commis et des employés des compagnies de commerce, y compris Louis Hébert, venu comme apothicaire du poste. La colonisation, qui débute cette année-là, amène des soldats pour le fort et des artisans pour le travail. Les premiers colons agricoles ne commencent d'arriver que l'année suivante et ce sont eux qui, - censitaires, laboureurs ou journaliers, - recrutés par la Compagnie de la NouvelleFrance, les seigneurs et les communautés religieuses, au cours de la période, formeront la majorité des émigrants qui osent s'expatrier. Avec eux arriveront, selon les besoins et les demandes, des hommes de métier, charpentiers, maçons, serruriers, armuriers et boulangers. A elle seule, la Société de Montréal embauchera environ quatre cents colons, artisans et soldats pour sa seigneurie. La majorité de ces habitants, comme on appelait les émigrants établis en permanence au pays, pour les distinguer des hivernants, étaient passés au Canada à titre d'engagés, c'est-à-dire à la suite d'un engagement de servir trois ans à salaire fixe, à l'expiration duquel ils devenaient libres de prendre une terre ou d'exercer un métier ou de rentrer en France, ou de travailler à leur gré comme volontaires sans attache quelconque.

Se superposant à ce fonds démographique, dont ils ont plus d'une fois suscité ou encouragé l'émigration, vinrent successivement s'installer au pays quelques gentilshommes plus ou moins impécunieux, comme de Repentigny, de Tilly, La Potherie et Le Neuf du Hérisson; des animateurs, comme Giffard, Denis et Chavigny; des fonctionnaires, comme Juchereau, Sevestre et Lotbinière; et, se haussant par leurs qualités au palier supérieur, des soldats, comme Bourdon et Villeray, et des interprètes, comme Godefroy et Boucher. La majorité d'entre eux furent gratifiés par les Cent-Associés de seigneuries pour lesquelles ils recrutèrent, selon leurs moyens, quelque nombre de colons. Avec les Pères jésuites, les prêtres séculiers et les Messieurs de Saint-Sulpice, ces divers éléments composaient la classe dirigeante du pays. 
Toute cette population ne comprenait, en principe, que des catholiques, l'édit de 1628 excluant les huguenots de la NouvelleFrance. Cependant, il se rencontra toujours quelques réformés dans le pays, la plupart venus à bord des vaisseaux de La Rochelle. Il s'en trouvait avec Champlain en 1633. D'habitude, ils ne faisaient que de brefs séjours, mais plusieurs restèrent dans la colonie à titre de matelots, de commis et même de colons, dont les Jésuites s'empressaient de poursuivre la conversion. Ainsi, on peut relever des abjurations, l'une en 1635 , deux en 1637 , celle de Champigny en 1646, qui devint le chantre recherché de la paroisse. En 1653, chose étonnante, les deux fondateurs de la VilleMarie mystique, La Dauversière et Maisonneuve, à court d'effectifs, recrutèrent des huguenots pour le poste Montréaliste, dont plusieurs renoncèrent plus tard au calvinisme. En quatre ans, sous Mgr de Laval, eurent lieu cinq abjurations, dont celles de trois femmes, qui se marièrent au pays. Ce ne sont là que des présences particulières, que la chronique a notées, mais elles suffisent à indiquer que des huguenots entraient au pays, et que la plupart de ceux qui s'y fixèrent finirent par se ranger au catholicisme. Il n'exista donc pas d'exclusion totale.

Venus de dix provinces principales, quel témoignage l'histoire a-t-elle laissé de la qualité morale de ces émigrants, en face de certaines rumeurs qu'il passait au pays « quantité de garnements, et des filles mal vivantes ?» Ce vague racontar est né de la confusion dans le public de l'époque entre les enrôlements pour les « isles » des Antilles, - Saint-Christophe, la Guadeloupe, la Martinique, Saint-Domingue et Cayenne, - qui acceptaient «toute sorte de gens ramassez » n'importe où, et les embarquements pour les «isles de terre ferme de Canada. » Devant les nouvelles à la main relatant «l'Adieu des Macquereaux et Macquerelles allant ... aux Isles, » les contemporains n'établissaient aucune distinction entre les différentes colonies, toutes désignées également sous le nom des «isles. » Mais «ce faux bruit», selon le terme d'un Père jésuite, ne résiste pas à l'examen des faits et des documents. Il suffit de rappeler que nombre des premiers émigrants furent recrutés par les Cent-Associés qui, ne recherchant pas le profit, se préoccupaient surtout «que Dieu soit 
servy fidèlement », et que la plupart des autres furent choisis par les seigneurs et les communautés religieuses dont le caractère et l'intérêt exigeaient l'esprit de travail et les bonnes mœurs de leurs engagés. Enfin, les Jésuites, qui guidaient et surveillaient toute la vie sociale de la colonie, ne cessaient de réclamer «des jeunes hommes forts, vaillants et courageux, mais surtout singulièrement vertueux ». Ils exerçaient une telle surveillance de l'immigration qu'ils disent avoir, à l'occasion «vu», donc visité, tous les vaisseaux qui arrivaient de France. Si, malgré tout, il se glissait au pays quelques "garnements, écrit le meilleur témoin de l'époque, Pierre Boucher, c'est qu'on ne les connaist pas », et de plus, «éclairez de trop prés», sont-ils « obligés de vivre en honnestes gens, ou ne tardent-ils pas à repartir »; car la longueur de l'hiver et le voisinage des Iroquois découragent vite le fainéant, l'aventurier ou le mécréant. Quant à ceux qui font scandale, on a vite fait de les expulser, tout considérables qu'ils peuvent être, comme le capitaine La Barre, de Montréal. Parfois, on exagère même la sévérité: ainsi Courville est arrêté et embarqué pour la France par simple crainte du « rapt imminent » de haute demoiselle d'Auteuil, qui, trop sensible aux agréments de ce beau roturier, est mise en sûreté à Beauport, chez M. Giffard. Les faits et les documents s'accordent dans l'affirmation que ces colons d'avant 1663 sont presque tous de race saine, de stricte honnêteté, d'esprit laborieux et de solide dévotion.

Quant aux filles émigrantes, - deux cent vingt-huit de 1654 à 1663 , - les quatre septièmes appartiennent à la classe rurale, deux septièmes descendent de familles urbaines et le dernier septième sont filles de petite noblesse ou de bonne bourgeoisie. Elles passent au pays, les unes attirées par des parents ou des émigrants de leur région, les autres recrutées par des institutions religieuses. Elles arrivent par petits groupes de trois, quatre ou cinq. Les groupes plus nombreux voyagent sous la conduite d'une religieuse ou de Jeanne Mance ou de Marguerite Bourgeoys. En 1641 , le $P$. Le Jeune écrira qu'il a « vu tous les vaisseaux » venus de France et que «pas un n'estait chargée » que d'excellentes sujettes. En 1654, le P. Le Mercier déclarera qu'on n'admet que «des filles fort honnestes » et « point d'autre dans cette nouvelle 
peuplade», avec défense "aux vaisseaux d'amener de ces marchandises de contrebande » féminine. Enfin, en 1662, Boucher ajoutera qu'avant l'embarquement, «il faut qu'il y ait quelques uns de leurs parents ou amis qui assurent qu'elles ont toujours esté sages. » Car les autorités civiles et religieuses sont rigides sur le point des mours. Quand, en 1650, deux femmes s'oublient au point de faire les «vilaines», elles sont bannies après fustigation publique et, quand une brebis galeuse réussit, en 1658, à se glisser à Québec, le gouverneur d'Argenson la déporte aux frais de l'armateur complice. Contrairement aux rumeurs, la Nouvelle-France ne reçut aucune fille publique et son émigration féminine au contraire, se révèle soigneusement surveillée et exceptionnellement saine.

D'où vient alors la légende de sa mauvaise réputation. Il est arrivé qu'à cette époque, s'amorçait la colonisation des Antilles, où, devant la forte majorité des émigrants célibataires, s'introduisit la pratique d'expédier des épouseuses dans les îles, qu'on se disputait à prix d'argent. L'appât du gain s'y mêlant, des armateurs n'hésitèrent pas à racoler pour le voyage des filles de mœurs douteuses et même des femmes publiques. Comme l'ont expliqué dans le temps le gouverneur d'Argenson et Pierre Boucher au public mal informé, ignorant la géographie et ne distinguant pas entre les colonies, il suffisait que le Canada fût une terre d'Amérique pour en conclure aussitôt qu'il servait comme «les Isles de Saint-Christophe et la Martinique » d'exutoire aux courtisanes et aux mauvaises filles de Paris.

Cependant, tout en reconnaissant la belle santé morale de la première génération canadienne, il ne faudrait pas se laisser induire en erreur par la propagande des Relations, affirmant que c'est « la résolution de se donner à Dieu, » qui a provoqué l'émigration des colons de la Nouvelle-France et qu'on trouve «en tous un désir ardent de leur salut, et une estude particulière de la vertu ». Il faut également se garder des outrances de la Sœur Morin, dépeignant par ouï-dire, Montréal comme « une image de la primitive église ». Omettant ces pieux embellissements, l'histoire établit que la plupart de ces émigrants sont venus sous con- 
trat de travail, gagnés par l'attrait d'une vie plus libre et d'une amélioration de leur sort.

Inévitablement, il s'est trouvé parmi eux, selon les termes des Jésuites, des âmes «basses » et des «esprits plus libertins 》 et, selon Boucher, de véritables «garnements », sans qu'il fût possible de les connaître. A telle enseigne que le chevalet se dressait sur la grande place de Québec, dès 1633, où l'on attachait, à l'occasion, des ivrognes, des blasphémateurs et d'autres délinquants. En 1654, deux «vilaines »y sont fouettées publiquement. Montréal prononce la première sentence de mort en 1648 et, l'année suivante, Québec assiste à la première exécution capitale et bientôt à une deuxième, alors que la colonie ne réunit pas cinq cents âmes. D'autres suivront au cours des années. Aux Trois-Rivières, où quelques-uns boivent, jurent plus que de raison et se battent en duel, on voit même d'authentiques gentilshommes, comme les frères Le Neuf, assaillir des manants à coup de poing, pendant qu'à Montréal, des femmes s'invectivent ou vont jusqu'à bâtonner des soldats. Dans la région, la plus populeuse de Québec, la chronique signale des rixes, des duels, des meurtres et des crimes d'incendie. D'ailleurs, chaque poste possède sa petite prison et Boucher proclame qu'on «scait aussi bien pendre en ce pays-là qu'ailleurs. » Mais l'existence de ces inévitables déchets d'humanité n'enlève rien aux remarquables qualités des neuf dixièmes de la population, hommes probes et travailleurs, femmes industrieuses et dévouées, pratiquant une solide piété et faisant preuve d'irréductible vaillance et de véritable héroïsme aux heures des pires dangers. Surtout, la moralité s'y maintient à la hauteur de la vertu: la conduite des femmes demeure irréprochable au point que, de 1621 à 1661, la statistique ne relève qu'une seule naissance en dehors de la légitimité.

Contrairement à ce qu'on a prétendu, cette population ne pratique pas l'égalitarisme. Sortie d'une France hiérarchisée, elle reconnaît en gros trois classes: les principaux, les médiocres et le commun, où chacun « doibt estre eu égard à sa naissance, ses facultés, sa capacité et sa famille. »Les Jésuites, en 1645, divisent leurs invités en « quatre départements » ou catégories, qu'ils reçoivent en des salles distinctes. La prétention s'insinue par- 
tout: les fidèles se disputent la préséance dans l'offrande du pain bénit, «chacun s'estimant plus qu'il n'est ». Madame Marsolet, épouse d'un interprète devenu seigneur, présente le pain bénit avec «plus d'appereil » que le gouverneur, ornant son panier «de bouillons de gaze » et de cierges. Si la Nouvelle-France ne peut batailler autour des tabourets de la Cour, elle connaît maintes querelles de préséance. L'évêque Laval s'irrite de voir donner la première place au gouverneur d'Argenson aux cérémonies civiles, et fait un jour enlever du chœur le fauteuil de son rival. "Les gentilshommes ou soy disant tels » repoussent les marguilliers au second rang des processions, que l'évêque supprime en représailles; les conseillers demandent de recevoir les rameaux de la main d'un ecclésiastique, et refusent « d'aller à l'adoration de la croix en la manière que faict le commun peuple »; à leur tour, les officiers de la Justice disputent aux conseillers le droit de marcher les premiers à la procession. Même les Hospitalières réclament contre les Ursulines, en 1646, le droit au premier reposoir de la Fête-Dieu.

Ces querelles naturellement se déroulent dans la petite capitale de Québec, qui seule réunit les dirigeants de l'État, de l'Église et des affaires. Sa modeste population de quelques centaines d'âmes, mêlant à sa dévotion le goût des rencontres et des conversations, afflue chaque fois aux cérémonies religieuses, auxquelles les Jésuites donnent tout l'agrément et la solennité possibles dans ce poste perdu. Au début, les violes, à défaut d'orgue, font entendre leur frêle musique; plus tard, on recrute un chœur qui peut chanter en quatre parties aux messes hautes le dimanche. Dans la nouvelle église, après 1650 , qui possède un orgue, on célèbre les cérémonies avec diacre et sous-diacre. A Noël, à la messe de minuit, malgré le froid, qui oblige de mettre un réchaud sur l'autel, l'église suffit à peine à contenir la foule des fidèles. Les feux de la Saint-Joseph et de la Saint-Jean, auxquels préside le gouverneur et qui s'accompagnent de la bénédiction du bûcher et du chant du Te Deum, deviennent des événements de l'année.

Parfois, grandes distractions, surviennent des représentations théâtrales, initiative remarquable dans un poste de quelque centaines de personnes. Dès 1640 , se joue une tragi-comédie, 
où triomphent Martial Piraube, secrétaire du gouverneur, et de «bons acteurs ». En 1646, on se hausse au Cid de Corneille, à Héraclius, en 1651, et de nouveau au Cid, l'année suivante. Le premier ballet se donne à une noce, en 1646, suivi d'un second, l'année qui suit, dans le magasin de la compagnie. Au collège des Jésuites, on accueille en public le gouverneur de Lauson par un discours latin et des vers français, et son successeur d'Argenson est régalé d'un petit drame en français, en algonquin et en huron, et Mgr de Laval est l'hôte d'honneur d'une représentation dans la chapelle de Québec, où «tout alla bien ».

Les mariages naturellement forment l'occasion d'exceptionnelles réjouissances, où les violons président aux danses, pour la première fois en 1645. Les noces du sénéchal, fils du gouverneur Lauson, en 1651, durèrent trois jours avec grands dîners, où se trouva chaque fois un Père jésuite. On se marie très tôt, dès qu'une fille est nubile ou qu'une épouseuse arrive de France, et les enfants viennent nombreux «bien faits, grands et robustes 》, avec un "esprit assez bon, mais un peu libertins », c'est-à-dire qu'on a «de la peine à les captiver pour les estudes», soit à l'école de Montréal, au couvent des Ursulines ou au collège des Jésuites à Québec. L'événement mondain de l'année reste, s'ouvrant au bruit du canon, le jour de l'An. La garnison salue le gouverneur d'une salve d'arquebuses et les habitants viennent lui offrir leurs hommages. Toute la journée, l'on échange des visites et des cadeaux. Ainsi, le gouverneur envoie aux Jésuites des bouteilles de vin d'Espagne et un coq d'Inde, ou des pigeons et des chapons. Ces derniers lui offrent en retour une croix de relique, à sa femme, "Mademoiselle la Gouvernante », un petit livre. Les Pères font porter une couple de bouquets de fleurs aux Ursulines et aux Hospitalières. Celles-ci répondent par des chapelets, des médailles en reliquaire, des gâteaux, des chapons ou de «belles pièces de tourtières ». Les notables reçoivent des livres de piété et donnent des chandelles, des pruneaux, ou de l'hypocras, qui est du vin au sucre et à la cannelle. On échange encore des Agnus $D e i$ et des écorces de citron, des images pieuses et des pruneaux. Parmi le peuple les petits cadeaux sont aussi de mode, où figurent des «mitaines», des «souliers sauvages» et des mouchoirs. 
Ces habitants, qui, dès 1651, s'intitulent «Canadois », premier prodrome de nationalisme, de quoi vivent-ils ? Les artisans, de leur métier, et les plus nombreux, les censitaires, de leurs cultures, grains et légumes. Mais la grande ressource du pays reste la fourrure. Elle produit de deux à trois cent mille livres par an, plus ou moins, selon le prix du castor gras, qui oscille entre dix livres la livre pesant et treize livres, tandis que le castor neuf se vend à la moitié environ de ce prix. Aux sociétés détentrices du monopole, les bénéfices permettent de solder «toutes les charges » de la colonie et de réaliser des profits en temps normal. Quoique l'habitant ne touche pour son castor qu'environ la moitié du prix de vente en France, le troc lui procure quelque gain, mais il profite davantage aux quelques coureurs de bois, qui commencent ce métier après 1648. Cette fourrure, qui sert de monnaie d'échange, présente un grand avantage; elle épargne le vingtcinq pour cent de profit qu'on ajoute au prix des marchandises de France. Aussi les journaliers préfèrent-ils recevoir leur salaire en cette monnaie. Tout le monde l'utilise, les ecclésiastiques comme les civils, et même les Jésuites, à qui seuls, cependant, on reprochera d'en faire la traite. A côté de l'agriculture et de la pelleterie, les plus entreprenants tentent d'exploiter quelques ressources du pays. Dès 1648, Abraham Martin, le premier, inaugure la pêche aux loups-marins à Tadoussac et, l'année suivante, se forme une société dans le même dessein. En 1651, Godefroy, Martin et Grouvel ont chacun une barque pour le commerce d'outre-atlantique. En 1653, part de Québec un chargement de bois pour la France. Cette même année, d'Ailleboust et Jean Paul Godefroy s'associent à Jean Bourdon pour la pêche à la morue, que leur vaisseau le Saint-Jean doit écouler aux Antilles contre des produits des îles qu'il ira échanger contre des marchandises de France, qu'il embarquera pour Québec, audacieuse initiative de commerce triangulaire que reprendra vingt ans plus tard l'intendant Talon. Quelques chefs poursuivent la recherche de mines de cuivre, d'antimoine et de plomb, ou s'intéressent à la présence de magnifiques chênaies. On le voit, malgré sa faible population et ses modestes moyens, la colonie avait déjà pleinement saisi les possibilités économiques du pays et se préoccupait 
de leur exploitation: grains, pêcheries, forêts et minéraux, tout ce que décupleront les siècles suivants.

La question se présente, que Paris posait à Boucher: "Que coûte la vie en Nouvelle-France ?» Par suite du taux élevé du transport et des risques maritimes, les marchandises indispensables, vêtements, chaussures, ustensiles, outils, vins, eaux-de-vie, armes, poudre, balles, «et une infinité de petites commodités», comme les articles de mercerie, subissent à leur entrée au pays une majoration de vingt-cinq pour cent. Cette hausse se transmet automatiquement aux denrées et aux services dans la colonie. Comme résultat la vie coûte à Québec deux à trois fois plus cher qu'à Paris. La livre de beurre qui se paie cinq sous en France, s'élève à douze au pays; les charpentiers et maçons français ne touchent que quinze sous quand leurs confrères canadiens en reçoivent quarante; le manœuvre de province se contente de dix sous par jour, mais celui de Québec, étant nourri, en réclame trente en été, vingt en hiver; le vêtement se vend au double du prix de France. Voici quelques prix canadiens de l'époque: le minot de blé de maïs ou de pois, de 5 à 8 livres; la barrique de cinq cents anguilles, 25 livres; une vache, 120 livres; un cochon, 45 livres; la corde de bois, 10 sous. Enfin la nourriture d'un homme de travail atteint 10 sous et plus par jour. Le vin coûtait dix sous la pinte et l'eau-de-vie, trente. La livre ou franc valait vingt sous.

Quant à la structure politique de la colonie, s'adaptant aux circonstances du lieu, elle est, au début, d'une extrême simplicité. Sous dépendance royale, la Compagnie de la Nouvelle-France possède le pays en pleine suzeraineté avec monopole de tout le commerce. Désigné par elle, mais recevant sa commission du roi, un gouverneur exerce toute l'autorité civile et militaire, y compris le droit de nommer des officiers de justice ad hoc et de juger en dernier ressort. A ses côtés, le commis général de la Compagnie dirige les opérations de la traite et la gestion des finances. Après 1645, il est remplacé par le commis général de la Communauté jusqu'en 1647 , alors que s'opère une curieuse coexistence de juridictions. Le roi règle et modifie à sa guise le régime admi- 
nistratif; la Compagnie des Cent-Associés concède les terres, nomme les officiers de justice et détient un contrôle des opérations de la traite des fourrures; la Communauté, par le Conseil de Québec, exploite et gère ce troc et tout le commerce, ainsi que la finance du pays. Le gouverneur garde cependant, au-dessus du conseil, une autorité suprême et finale, soumise à la seule décision royale. Quant au peuple, il obtient successivement, le droit d'élire des syndics, d'élire des représentants au conseil et finalement d'élire des conseillers, qui régissent le commerce et la finance. Ces pouvoirs administratifs, il importe de le signaler, ces délégués du peuple les exercent non pas à titre de régnicoles, mais à titre de représentants d'une société de commerce qui est la Communauté des habitants. En tant que citoyens, les Canadiens ne pouvaient qu'élire des syndics veillant au bien de leur groupe respectif, présenter des « remontrances » au gouverneur, au Conseil de Québec et finalement au roi lui-même par voie de mémoires ou de délégations. Il est à noter que, sans autorisation préalable du souverain, Québec entame directement avec les colonies américaines des négociations pour la conclusion d'une alliance militaire et commerciale, droit que le Canada contemporain n'obtiendra qu'en 1926. Au début, dans le domaine judiciaire, vu la minime population et l'absence de cours régulières, les causes se plaidaient devant un juge et un greffier ad hoc, et très souvent devant le gouverneur de Québec ou celui des Trois-Rivières avec appel au premier. Montréal se contentait d'une cour seigneuriale, comme le feront deux ou trois seigneuries. En 1651, Lauson établit une magistrature à Québec et aux Trois-Rivières, dont les appels relèvent du gouverneur et, après 1659 , du Parlement de Paris pour les causes importantes.

Plus que cette structure administrative, ce qui préoccupait avant tout le colon, c'était le régime foncier. Ce dernier ne pouvait que reproduire celui de la mère-patrie et transférer en Amérique la féodalité, mais la féodalité évoluée du dix-septième, qui s'adaptant aux conditions d'un pays de haute futaie en pleine sauvagerie, réduit au minimum les exigences des diverses tenures. La Compagnie de la Nouvelle-France ne concède pas de fiefs nobles, qui auraient requis la sanction royale, mais des 
seigneuries comportant la simple obligation de les peupler et de les défricher. Leurs propriétaires, de leur côté, s'emploient, selon leurs moyens, à recruter des colons, à qui ils cèdent en censive, des étendues de terre de trente à quarante arpents de profondeur sur trois ou quatre de front généralement en bordure du fleuve ou d'une rivière, seules routes de communication. Ces censitaires ne payent qu'un cens modique, qui va d'un denier à un sou par arpent, ainsi qu'une rente qui varie de dix à vingt sous par arpent de front. Avec sa terre, l'habitant a droit de pêche sur sa devanture et de chasse dans sa concession, avec faculté d'obtenir du gouverneur un permis de pêcher et de chasser ailleurs. De son côté, outre des emplacements de maison autour de leurs forts, la Compagnie concède directement à des colons des terres en censive dans les banlieues de Québec et des TroisRivières.

Ces émigrants, habitués au pain noir dans une France qui les rabaisse et les opprime, s'acclimatent aussitôt avec contentement dans un pays sans taille ni corvées ni gabelle, où ils possèdent en toute propriété contre de minimes redevances une terre libre et franche, qui, en quelques années, leur fournit «abondamment » le blé, les fruits et les légumes, tandis que la forêt leur offre le bois nécessaire à leur maison, à leur mobilier et à leur chauffage. De plus, cette terre canadienne récompense largement son propriétaire. Dès 1643 , la plupart des colons récoltent froment, seigle, orge et foin, récolte qui s'augmente d'année en année, si bien qu'en 1653, la colonie pouvait « en cela nous passer des secours de France, quelque nombre que nous soyons ici». D'un bon rendement dans la région de Québec, la terre accuse une fertilité plus grande aux Trois-Rivières, véritable grenier à blé aux jours de disette, mais la plus excellente est celle de Montréal qui, plus au sud, produit de riches moissons et des fruits et légumes d'une saveur particulière. Le bétail, bœufs, vaches et porcs, se multiplie partout, ainsi que les poules, les dindes et les pigeons de France.

Cependant, à côté du gros gibier, élans et orignaux, et des oiseaux qui foisonnent, surtout les «tourtes», ces pigeons sauvages qu'on abat par centaines, la manne du pays, c'est l'anguille. 
Après la pêche abondante du saumon, en août, que suivait celle de l'esturgeon, descendaient en septembre, du lac Ontario, les bancs voyageurs d'innombrables anguilles et pendant deux mois, leur pêche s'activait. Le total annuel des prises se chiffraient entre quarante et soixante-et-dix mille. " D'une excellente garde, soit séchée au feu, soit salée», de cette anguille, «beaucoup meilleure » que celle de France, on faisait provision abondamment pour toute l'année. Enfin, la liberté de troquer la fourrure rapportait quelque profit, qui permettait de s'offrir certaines douceurs, entre autres, le «petit coup» d'alcool, ou le verre de bière de Québec, et parfois même du vin de France. Mais beaucoup se contentaient de «bouillon», breuvage qu'on fabriquait avec de la pâte fermentée et de l'eau épicée.

Aussi, dans ce pays sain et salubre, même durant les cinq mois de bel hiver, lumineux et tonifiant, où, en moins de quatre ou cinq ans, chacun peut être à son «aise » et «bien ajustez » pour sa condition, l'émigrant s'enracine-t-il avec une joyeuse satisfaction. Il habite une petite maison plutôt de bois que de pierre, que coiffe en pente un toit de planches. De petites fenêtres carrées encadrent des chassis garnis, en guise de vitres, de forte toile ou de papier huilé. Les meubles se réduisent à une table, des lits, des coffres à linge et quelques sièges rustiques. Dans la cuisine, pièce principale du logis, domine une large cheminée propre aux grandes flambées de l'hiver, où se cuisent les plats à la crémaillère ou sur trépieds. On s'éclaire à la chandelle; pour cette raison, on se couche très tôt et on se lève aux premières lueurs du soleil. Il arrive même que des messes se disent à quatre heures et des mariages se célèbrent à cinq heures du matin. Propriétaire de sa terre, féconde en grains, avec le jardin, où sa femme cultive de beaux légumes et, dans un coin, quelques roses et force œillets, mettant souvent le gibier au pot et mangeant du pain blanc, le colon s'éprend à ce point de ce «Paradis terrestre» qu'il rejettera toute idée de le quitter, même aux jours les plus sombres de la guérilla iroquoise.

Gustave LANCTOT 